\title{
Report of the forth Asian Prostate Cancer (A-CaP) study meeting
}

\author{
Ji Youl Lee ${ }^{1}$, Tomotaka Taniguchi ${ }^{2}$, Kai Zhang ${ }^{3}$, Chi Fai $\mathrm{Ng}^{4,5,6}$, \\ Lukman Hakim ${ }^{7}$, Rainy Umbas ${ }^{8}$, Choung-Soo Kim ${ }^{9}$, Byung Ha Chung ${ }^{10}$, \\ Wun-Jae Kim ${ }^{11}$, Teng Aik Ong ${ }^{12}$, Jasmine Lim ${ }^{12}$, \\ and Hideyuki Akaza ${ }^{13,14, * 15}$
}

\begin{abstract}
'Department of Urology, St. Mary's Hospital, The Catholic University of Korea, Seoul, South Korea, ${ }^{2}$ Department of Strategic Investigation on Comprehensive Cancer Network, Graduate School of Interdisciplinary Information Studies, University of Tokyo, Tokyo, Japan, ${ }^{3}$ Beijing United Family Hospital and Clinics, Beijing, China, ${ }^{4} \mathrm{SH}$ Ho Urology Centre, The Chinese University of Hong Kong, Hong Kong, China, ${ }^{5}$ Robotic Surgical System Training Programme, Jockey Club Minimally Invasive Surgical Skills Center, The Chinese University of Hong Kong, Hong Kong, China, ${ }^{6}$ Division of Urology, Department of Surgery, The Chinese University of Hong Kong, Hong Kong, China, ${ }^{7}$ Department of Urology, Airlangga University/Dr Soetomo General Hospital of Surabaya, Surabaya, East Java, Indonesia, ${ }^{8}$ Department of Urology, Dr Cipto Mangunkusumo Hospital/Faculty of Medicine, University of Indonesia, Depok City, West Java, Indonesia, ${ }^{9}$ Department of Urology, Asan Medical Center, University of Ulsan College of Medicine, Seoul, South Korea, ${ }^{10}$ Department of Urology Gangnam Severance Hospital, Yonsei University College of Medicine, "Department of Urology, Chugbuk National Medical University Hospital, ${ }^{12}$ Department of Surgery, Faculty of Medicine, University of Malaya, Kuala Lumpur, Malaysia, ${ }^{13}$ Department of Strategic Investigation on Comprehensive Cancer Network, Graduate School of Interdisciplinary Information Studies, University of Tokyo, Tokyo, Japan, and ${ }^{14}$ Emeritus Professor, University of Tsukuba, Tsukuba, Japan
\end{abstract}

*For reprints and all correspondence: Hideyuki Akaza, Department of Strategic Investigation on Comprehensive Cancer Network, Graduate School of Interdisciplinary Information Studies, University of Tokyo, 4-6-1, Komaba, Meguro-Ku, Tokyo 153-8904, Japan. E-mail: akazah@siccn.org

${ }^{15}$ Other authors are listed in Appendix of last page.

Received 12 March 2019; Editorial Decision 19 March 2019; Accepted 5 April 2019

\begin{abstract}
The Asian Prostate Cancer (A-CaP) Study is an Asia-wide prospective registry study for surveying the treatment outcome of prostate cancer patients who have received a histopathological diagnosis. The study aims to clarify the clinical situation for prostate cancer in Asia and use the outcomes for the purposes of international comparison. Following the first meeting in Tokyo on December 2015, the second meeting in Seoul, Korea 2016, the third meeting in Chiang Mai, Thailand, on October 2017, the fourth meeting was held in Seoul, again on August 2018 with the participation of members and collaborators from 13 countries and regions. In the meeting, participating countries and regions presented the current status of data collection and the A-CaP office presented a preliminary analysis of the registered cases received from each country and region. Participants discussed ongoing challenges relating to data cleaning and data updating which is the next step of the A-CaP study following the data collection phase between 2016 and 2018. There was specific difference in term of the patient characteristics, and initial treatment pattern among East Asia, Southeast Asia and Turkey, and Jordan. Finally, a close relationship between prevalence of PSA test and disease stage of the patients at diagnosis in Japan and Malaysia was discussed.
\end{abstract}

Key words: Asia, cancer, database, registry, prostate cancer, A-CaP, J-CaP 


\section{Introduction}

The forth meeting of the Asian Prostate Cancer (A-CaP) Study was held on 31 August 2018, in Seoul, Korea, in conjunction with the 2018 Korea Prostate Society (KPS) and Asia Pacific Society of Uro-oncology (APSU) Joint International Symposium. The meeting was attended by representatives of 13 countries and regions in Asia participating in the study (China, Hong Kong, Indonesia, Japan, Jordan, Korea, Malaysia, Philippines, Singapore, Taiwan, Thailand, Turkey, Vietnam) and collaborators from the USA. Following on from the A-CaP launch symposium in December 2015 and the second A-CaP meeting in Seoul, Korea in September 2016, the third meeting in Chiang Mai, Thailand in October 2017, members discussed the current status of progress with patient registration, background factors, the outlook for further registrations, and ways in which to utilize the database that is being accumulated through the A-CaP project(1-3). In the business meeting held prior to the scientific meeting the A-CaP office also mentioned the present status of data collection and way to go, and data follow-up method. The following is a summary of the proceedings of the Forth A-CaP Study Meeting.

\section{Opening}

W.-J.K. (Chungbuk National Medical University Hospital) expressed his tremendous anticipation that in the future the A-CaP study would help to promote and share knowledge on prostate cancer in Asia. He invited all members to provide their annual report.

\section{Year report from each A-CaP member country}

\section{Japan}

Mizuki Onozawa (International University of Health and Welfare) noted that the number of patients registered is steadily increasing, reaching 11000 in July 2018, spread over 140 institutions in Japan. The median number of patients per institution is 70 .

In terms of age distribution the median age is 72 . The Gleason score has increased in comparison to previous studies. The proportion of T3 and T4 cancer is stable for the 3 years of the study and the proportion of $\mathrm{T} 2$ has increased, which may be due to staging accuracy. Stage IV disease is stable at $15 \%$ and the J-CAPRA scores are also stable.

In terms of initial treatment, the proportion of ADT is gradually decreasing and the proportion of prostatectomy is increasing $(\sim 34 \%)$.

In terms of prostatectomy, most of these are done robotically. Curative radiation is mainly implemented using IMRT/IGRT. Particle therapy is still only a small percentage of therapy, it has just been approved for insurance coverage in Japan and it may therefore increase in the future.

In terms of initial medical/surgical castration therapy, Leuprolide accounts for the greatest majority of therapy. The proportion remains unchanged over the last few years. Degarelix is the preferred therapy in J-CAPRA high score cases.

In terms of the count of PSA data per patient, there is insufficient follow-up data. The majority of patients in the A-CaP study only have baseline PSA data available.

In terms of survival data, only 59 deaths have been reported among 5401 patients, but the number of follow-ups is still low.

In summary, it can be said that although the number of newly registered patients is increasing, background factors seem to be almost unchanged or slightly worse in comparison to previous J-
CaP data (2010). It should also be noted that clinical practice patterns are changing dramatically and more follow-up data will be required.

\section{Discussion}

W.-J.K. asked about robotic surgery and whether it is covered by insurance. Yoshihiko Hirao (Osaka Gyoumeikan Hospital) responded that in Japan every form of treatment is covered by health insurance, including brachytherapy and proton therapy.

Matthew Cooperberg (UCSF) noted that in the previous J-CaP study there was a remarkable number of patients registered. He asked if recruitment and registration had been more difficult for the A-CaP study. Dr Onozawa responded that the main difference is that in the previous $\mathrm{J}$-CaP study only patients receiving ADT were registered, whereas for the A-CaP study all patients are being registered, regardless of the type of treatment.

C.S. Kim (Asan Medical Center) asked why prostate cancer is increasing in Japan. Dr Onozawa responded that the aging of the population may be one of the reasons.

T.A. Ong (University of Malaya) asked about ADT and the use of Degarelix, asking whether this was a monthly injection or 3monthly injection. Dr Akaza responded that in Japan the monthly injection is the only type currently approved.

\section{Korea}

Seung Hwan Lee (Yonsei University) noted that the trend of prostate cancer incidence has been increasing year-by-year, with 10000 people being newly diagnosed each year. Prostate cancer is the fifth most common cancer in Korea, with 25.4 people per 100000 population being diagnosed. The 5 -year survival rate has been increasing in recent years, due to the effect of widespread PSA screening and early detection. In terms of the staging of the A-CaP study registered patients, the most common T stage is T2 (46\%). As expected, 90\% of patients are N0M0. The initial treatment in Korea that is most popular is radical prostatectomy $(65.4 \%)$.

In terms of registration for $\mathrm{A}-\mathrm{CaP}$, the volume of eligible patients' data is increasing, from 9 hospitals last year to 13 hospitals this year. In Korea, localized prostate cancer accounted for the largest number of newly diagnosed patients and most of them were treated with radical prostatectomy (RP). To enhance the study in Korea it will be necessary to secure more data collection personnel.

\section{Discussion}

H.A. asked about the number of patients undergoing surgery, which appears to be a very high figure. Dr Lee responded that the A-CaP study includes mostly large medical centers and screening has resulted in relatively younger patients being diagnosed, which is the reason for the higher proportion of RP. Dr Akaza suggested that it may be interesting to compare the proportion of RP and ADT.

Dr Hirao noted that T1 incidence is decreasing and T2 is increasing. He asked whether diagnosis is based on MRI or examination. Dr Lee responded that nearly all patients were examined by MRI.

\section{Taiwan}

Tong-lin Wu (Kaohsiung Veterans General Hospital) reported that only two hospitals in Taiwan have joined A-CaP and 500 patients have been registered. The median age of the registered patients is 72 . The $2 \%$ of patients have family history of prostate cancer. In terms of initial PSA $\sim 50 \%$ of patients had a level over 20 . The median level was 19.79. Image studies include whole body bone scan 
(93.2\%), MRI (79.6\%), CT scan (18.7\%) and PET scan (1.2\%). In terms of TNM staging $26 \%$ of patients have M+ and $18.3 \%$ of patients have $\mathrm{N}+$. In terms of initial treatment, but most popular treatment is RP $(34.1 \%), 86.5 \%$ of which are robot-assisted, which incurs out-of-pocket costs for patients. Curative radiation patients are 22.7 and $26.9 \%$ receive $\mathrm{ADT}+$ chemotherapy. The $15.5 \%$ of patients receive active surveillance.

In terms of the follow-up plan for patients in Taiwan, for organconfined disease the PSA is monitored every three months during the first 2 years and then every 6 months thereafter. A whole body bone scan is implemented when the PSA is rising. An MRI is arranged when PSA is rising and localized recurrence is suspected. For metastatic disease PSA is measured every 1-3 months and a whole body bone scan is performed when the PSA is rising.

\section{Discussion}

Dr Akaza asked whether PSA testing is covered by insurance. Dr Wu responded that PSA testing is covered by insurance. Dr Akaza asked about the national registration system for cancer. Dr Wu responded that there is a national registration system for 12 major cancers, including histological type and staging. Dr Wu recommended that it would be important to share a common language for follow-up studies in A-CaP in the future.

Mikio Namiki (Kanazawa University) asked about the relatively high level of patient burden and how treatment is determined. Dr Wu noted that the patient is consulted about the kind of treatment. If a patient cannot afford surgery, then they tend to receive radiotherapy, which is cheaper.

\section{China}

K.Z. (Peking University) noted that prostate cancer incidence is increasing rapidly in China, more than any other cancer. In terms of mortality, prostate cancer is also increasing. There are currently only 256 cases registered for the A-CaP study. Most cases received RP (207), followed by ADT (45). In terms of active surveillance, China is participating in the PRIAS study.

In terms of future plans, the main challenge in China is that there are no research nurses and only doctors are permitted to collect data, but many are too busy to collect data. In the future it is hoped that more doctors can be persuaded to join A-CaP. An app is being developed to facilitate follow-up data checks, reminding both the patient and the physician to implement follow-up checks.

\section{Discussion}

Edmund Chiong (National University Health System) asked about incidence and mortality and what are the contributing factors to the increase in prostate cancer in China. Dr Zhang noted that the increase in incidence and mortality can be attributed to the increase in PSA testing.

Dr Akaza asked about potential solutions for encouraging doctors to engage in data input. Dr Zhang responded that in smaller hospitals only doctors input data, so it is to be hoped that they can be persuaded to engage in data input in the future, or employ a person dedicated to data input operations.

Dr Akaza asked whether PSA testing is covered by insurance. Dr Zhang responded that most people in cities are covered by insurance, but coverage in rural areas is not as good.

Dr C.S. Kim asked why the number of robotic surgeries is so low. Dr Zhang responded that the reason is that there is still only a small number of robotic equipment available.

\section{Malaysia}

T.A.O. (University of Malaya) noted that with regard to the prostate cancer burden in Malaysia, incidence is 10.8 people per 100000 , with mortality of 4.6 per 100000 population. Since 2016 cases of prostate cancer have been registered in east and west Malaysia. Between January 2016 and May 2018 a total of 1306 new prostate cancer cases have been registered for the A-CaP study, covering most of the hospitals that provide urological service.

In terms of the demographic profile, Malays account for $38.7 \%$ and Chinese $43.8 \%$ of registered patients. The median age at diagnosis is 69.5 and the majority of patients have a PSA above 20. The majority of patients are T4, with $49 \%$ presenting with T4M1 disease.

In terms of the initial treatment, for Stage 1 cancers active surveillance/watchful waiting (ASWW) is the most popular form of treatment, whereas for Stage II and III cancers curative radiation is most often used. ADT \pm chemotherapy is used for the majority of Stage IV cases.

In terms of budget expenditure, Malaysia was fortunate to receive money from both $\mathrm{J}-\mathrm{CaP}$ and the NIH Malaysia, which has helped to fund support efforts at hospitals that are contributing to the study. Dr Lim has engaged actively in site visits to participating centers.

\section{Discussion}

Dr Akaza asked for suggestions about obtaining responsible persons for engaging in follow-up. Dr Ong stressed the importance of acquiring funding and ensuring sufficient manpower in order to engage in effective follow-up.

R.U. (University of Indonesia) noted that $\sim 50 \%$ of new patients have localized disease, but only $10-15 \%$ of patients receive curative treatment. Dr Ong responded that some patients may not want to receive treatment and another reason may be because some patients are low risk.

Dr Chiong noted that managing data centrally is the ideal method. He asked about the funding from NIH Malaysia, and how outcomes are being reported. Dr Ong noted that funding is based on performance and feedback and data must be provided to NIH.

Dr Zhang asked about the reason why $43.8 \%$ of patients are Chinese, whereas Chinese account for only $20 \%$ of the population. Dr Ong responded that the registry is based on urban populations, where ethnic Chinese tend to be located.

Bannakij Lojanapiwat (Chiang Mai University) asked about PSA screening in Malaysia. Dr Ong noted that PSA is generally not adopted or encouraged.

\section{Indonesia}

L.H. (Airlangga University) reported that the A-CaP study in Indonesia started in April 2016 and 408 patients are currently registered from nine primary hospitals and 27 affiliated hospitals, with 420 application users. The majority of patients are 75 years or younger, with $71 \%$ having normal BMI. The $52 \%$ were high school graduates or lower, $57 \%$ lived in urban areas. The $57 \%$ had PSA $>20.55 \%$ had GS $8-10$.

In terms of clinical T staging $57 \%$ had cT2a or higher. The $83 \%$ were M0. In terms of imaging CT is used in $12 \%$, MRI in $7 \%$ and cone-scan in $35 \%$.

In terms of initial treatment only $10.4 \%$ of patients underwent $\mathrm{RP}$, with or without neo/adjuvant treatment. The $62 \%$ received 
$\mathrm{ADT}$ as main treatment and in total ADT is used in $74 \%$ of patients.

Radiation treatment is used with first-line curative intent in $18.9 \%$ of cases. It is used as an adjuvant treatment after surgery in $17 \%$ of cases.

In terms of ADT, it is provided as main treatment for $45.6 \%$ of cases. Surgical castration only is implemented in $26 \%$ of cases, while LHRH a/a + long-term a/a is used in $25.2 \%$ of cases.

In terms of follow-up, Indonesia will continue to optimize data collection until the end of the collection period on 31 December 2018. Efforts will be made to invite more affiliate hospitals and register more new cases. A progress meeting will be implemented once to twice a year to update data and engage in data cleaning before it is sent to the A-CaP secretariat.

\section{Discussion}

Dr Lojanapiwat asked about histological examination. Dr Lukman responded that imaging is not generally used, due to constraints in the national health insurance system. In the outpatient setting it is not easy to plan CT and MRI scans. Therefore, bone scans are usually used.

\section{Hong Kong}

N.C.F. (The Chinese University of Hong Kong) noted that data for A-CaP is collected from three out of nine hospitals, covering more than $40 \%$ of the population. A total of 716 cases have been registered. The most prevalent age group at diagnosis is $71-80$, although a large number present at 61-70.

In terms of PSA, more than half of patients have a PSA between 4 and 20 . The specimen is taken by prostate biopsy. Approximately $42 \%$ of patients have a GS in excess of $7.67 \%$ of patients receive MRI imaging. The majority of patients $(41 \%)$ are T1c. In terms of initial treatment, RP accounts for $27 \%$, followed by: RT $21 \%$, ADT $28 \%$, ASWW $21 \%$ and others $3 \%$.

In terms of major challenges for follow-up, there is delayed availability of data in the Hong Kong public hospital system. Another issue is that of workload as most of the data is collected by Dr Ng's staff (two research assistants). This presents challenges for maintaining follow-up data.

\section{Discussion}

Dr Ong asked about the percentage of patients treated in public hospitals. Dr Ng responded that $80 \%$ of patients are treated by government hospitals and that most patients return to the public system for follow-up.

\section{Jordan}

Ghazi M. Al Edwan (Jordan University Hospital) noted that Jordan University Hospital and the King Hussain Cancer Center are providing data for A-CaP. Prostate cancer is the second most common cancer in men worldwide and the second leading cause of cancer death in men in the western world. There is no PSA screening in Jordan at the moment.

In the registered data, $\sim 30 \%$ of patients have PSA between 4 and 10 and $20 \%$ have PSA between 10 and 20 and $40 \%$ have PSA $>20.87 \%$ are diagnosed by prostate biopsy. The $30 \%$ have GS of $2-6,35 \%$ have GS of 7 and $27 \%$ have GS of $8-10$.

In terms of staging half of patients are $\mathrm{T} 2$ and $30 \%$ are $\mathrm{T} 1$. The $70 \%$ of patients are diagnosed with metastatic disease, reflecting the late presentation of patients. The $65 \%$ of patients receive a CT scan and $70 \%$ of patients received a bone scan. MRI was done for $45 \%$ of patients. PET scans was not done for patients currently registered. Most of the patients were treated with RP or ADT \pm chemotherapy.

\section{Discussion}

Dr Chiong asked about metastatic disease and screening. Dr Edwan noted that there is no screening for early detection of prostate cancer in Jordan, which leads to the high level of metastatic disease.

Dr Akaza asked about screening activities. Dr Al Edwan responded that efforts are being made to raise awareness about prostate cancer and implement PSA testing from an early age. All cancers are covered by the national health system in Jordan and RP is provided under the health system.

\section{Turkey}

Levent Türkeri (Marmara University) noted that one of the major challenges in Turkey has been data input and there are four data managers who are working to input data. However, manpower is still insufficient for inputting data. The incidence of prostate cancer in Turkey is 36 per 100000 . PSA is commonly used in Turkey through word of mouth screening programs. The number of patients being diagnosed is therefore increasing.

In terms of age at diagnosis, the majority of patients are relatively young, around 65 years. The most prevalent PSA score is between 4 and 9. Pathological specimens are largely taken using biopsy, with most cancers being adenocarcinoma. The Gleason score is in line with the general patient population. The majority of patients are $\mathrm{T} 2$ at diagnosis. In terms of imaging, CT and bone scans are performed more than MRI.

The most popular treatment for local treatment is open RP. About $18 \%$ of patients have metastasis and these generally receive LHRH with flare protection.

The data that has been collected to date has some biases. One of the problems is that the number of cases of radiation therapy is very low and the reason is because the cases come from urological practice, rather than urological oncology. Due to patient privacy issues it has proven difficult to access information about radiotherapy. Efforts are being made to anonymize data, which will make it possible to acquire such data in the future.

\section{Thailand}

Dr Lojanapiwat reported that 407 patients are registered for the A$\mathrm{CaP}$ study. Most of patients are more than 65 years old. In terms of $\mathrm{T}$ category, $42 \%$ of cases are $\mathrm{T} 1 \mathrm{c}$ and $\mathrm{T} 2 \mathrm{c}$ accounts for $18 \%$. The $70 \%$ of cases are $\mathrm{N} 0$ and $82 \%$ are $\mathrm{M} 0$.

In terms of imaging, $32 \%$ of patients receive CT scans, $40 \%$ receive MRI and $77 \%$ receive bone scans. PET scans were used for $2 \%$ of patients.

In terms of initial treatment, the most popular was RP without neoadjuvant treatment $(61 \%)$. Approximately $20 \%$ of patients have ADT.

In terms of follow-up, data has been gathered for $52 \%$ of patients, but has yet to be recorded in the database. Among the follow-up patients $23 \%$ of patients have a PSA of $>4$.

The second follow-up (after 1 year) has been gathered for 32\% of patients.

The 407 cases for the A-CaP study have been recruited in four centers. Most patients have an age more than 65 years. Tissue diagnosis is mostly from TRUS biopsy. The $36 \%$ have PSA $<10$ and $11 \%$ have PSA more than 100 . The $34 \%$ have high GS, with $42 \%$ 
having T1C and 30\% T3/T4. CT/MRI and bone scan are the investigation tools for staging. The $52 \%$ of patients have follow-up date from 6 months after recruitment, with $37 \%$ having follow-up data at one year after recruitment, with most of the patients still receiving the same treatment.

\section{Discussion}

Dr Akaza asked about the location of the collection centers. Dr Bannakij responded that three centers are in Bangkok and one in Chiang Mai. This provides the opportunity to compare the situation between urban and rural areas. Dr Akaza asked whether it would be possible to increase the number of collection centers. Dr Lojanapiwat responded that the four major centers offer the best options for collecting data, but that he would continue to consider options for recruiting other centers.

\section{Singapore}

Edmund Chiong (National University Health System (NUHS)) reported that prostate cancer is the third most common cancer in men in Singapore, with incidence of 29.7 per 100000 persons. Incidence has been increasing over the past decades. The $30 \%$ of patients present with Stage IV metastatic disease. There are three participating hospitals in the A-CaP study in Singapore.

In terms of progress, data sharing agreement contracts have been concluded with hospitals. A contract agreement has also been concluded with A-CaP. Single multi-institution IRB application for all hospitals for prospective patients and informed consent are required and these have been approved. The platform for collection is REDCAP and all three hospitals have implemented REDCAP training. Each hospital retains its own database autonomy.

In terms of the data, a multi-site IRB was approved in August 2017. The data is prospective data and all patients have been histologically diagnosed with prostate cancer from 1 January 2016 onwards. Informed consent has been obtained from all patients.

The median age is 71 years. In terms of initial PSA, about onethird of patients have a PSA between 10 and 20. There is an even distribution of staging between T1, T2 and T3. Most patients undergo CT or MRI for staging. In terms of initial treatment, there is a good distribution between RP, curative radiation, ADT \pm chemotherapy and AS/WW.

In terms of challenges, limitations on recruitment rates are the Human Biomedical Research Act of Singapore. Another challenge is the funding of research assistants. An application has been made for a research grant, but the results are pending. Working is ongoing to harmonize data fields and central subject identification is in progress. Accurate follow-up and oversight are also challenges that will require attention.

\section{Discussion}

$\mathrm{Dr} \mathrm{Vu}$ asked whether foreign patients are included. Dr Chiong responded that foreign patients are not included in the study.

\section{Philippines}

Jason Letran (Cardinal Santos Medical Center) reported that a total of 412 patients are registered for the A-CaP study in the Philippines, most of whom have come from the private sector. A major challenge is to encourage participation from the public sector. $65 \%$ of patients are between 60 and $70.83 \%$ are from urban regions of the Philippines. The $82 \%$ of patients had a PSA between 4 and 20 . The majority of patients receive a diagnosis from prostate biopsy. The
$67 \%$ of patients have a Gleason Score of 6 or $7.48 \%$ of patients have T1 staging. Although there is not PSA screening in the Philippines, this group of patients is from private hospitals, which reflects the lower staging status. The $29 \%$ received a CT scan and $30 \%$ received an MRI for staging. The $72 \%$ received a bone scan. In terms of initial treatment, $57 \%$ received RP.

In terms of challenges for the A-CaP study, a major challenge will be to clean up the data received. A further issue is the promulgation of the data protection act in the Philippines, which will entail further measures in order to ensure that the Philippines can continue to participate in the study.

\section{Discussion}

Dr Ong asked about the percentage of people treated in the public and private sector. Dr Letran responded that if more data from the public sector could be acquired it would be likely that the data would be significantly different. Robotic prostatectomy is only available privately in the Philippines.

\section{Vietnam}

Vu Le Chuyen (Binh-dan Hospital) reported that prostate cancer is increasing in Vietnam. To participate in the A-CaP study, a variety of documents have been received and 261 documents are compatible with A-CaP. In terms of average PSA at diagnosis, the most prevalent is around 20. Most Gleason Scores are between 7 and 9. The majority of cases present with T2 and T3 stage cancer. More than $50 \%$ of patients have MRI and bone scans.

In terms of treatment, 110 cases received RP, while 140 cases received ADT. Robotic prostatectomy is increasing in Vietnam. ADT is usually provided as a primary treatment and as adjuvant treatment following prostatectomy in some cases.

In terms of follow-up, only $10-15 \%$ of patients have been followed up to date.

Challenges for Vietnam are to increase the number of registrations, by expanding the number of hospitals. Another challenge is to increase follow-up efforts.

\section{Future plan for A-CaP data}

J.L. (University of Malaya) introduced a 'Tentative Comparison of Data between Malaysia and Japan.' She noted that $56 \%$ of all prostate cancer cases in Malaysia are Stage IV, but this figure rises to 65 and $73 \%$ in Sabah and Sarawak, respectively.

In terms of the prostate cancer burden in Malaysia and Japan, whereas in Malaysia the incidence is 10.8 per 100000 population, the incidence in Japan is 30.4 per 100000 . Mortality is 4.6 per 100000 in Malaysia, compared with 5.0 per 100000 in Japan.

A multivariable analysis between Japanese and Malaysian prostate cancer patients showed that Malaysian patients are diagnosed at a younger age. In terms of staging the majority of Japanese patients are diagnosed at Stage I, which is the reverse of the case for Malaysia, with the majority of patients being diagnosed at Stage IV.

In terms of initial treatment, for localized disease Japanese institutions will opt for prostatectomy for the majority of stage I and II cases, whereas in Malaysia the first choice of treatment for Stage I cancer is active surveillance, and curative radiation for Stage II cancer.

An issue to address was the weighting of the J-CAPRA scoring system, and whether it could be usefully be applied to Malaysia. The J-CAPRA scoring system was applied for the Malaysian cohort, including Gleason score, PSA, $\mathrm{T}$ stage, $\mathrm{N}$ stage and $\mathrm{M}$ stage. Using 
the J-CAPRA risk category classification, four Stage IV cases and 297 Stage IV cases were classified in the low risk group and intermediate risk group, respectively, suggesting that the degree of risk is being underestimated. Dr Hinotsu modified the items in the J-CAPRA score and reimplemented data analysis, which suggested that it may be preferable to modify the J-CAPRA risk category for M1 patients.

\section{Discussion}

Dr Bannakij asked about the modification of the J-CAPRA scores. Dr Lim responded that what was changed were the cut-off points, rather than the staging for scoring itself. Dr Akaza noted that Dr Onozawa had also worked on this topic.

Dr Onozawa remarked that the modified J-CAPRA score had shown itself to be promising for measuring data and it would be possible to use the original and modified J-CAPRA scores in the future.

\section{Comments from USA}

Dr Cooperberg noted that it is amazing how the A-CaP study has developed. He noted that it is now necessary to consider the following question: 'What will A-CaP answer?' This question will help to focus data collection efforts. The next discussion to have is to whether to concentrate only on disease present at diagnosis, but if further data is required, the importance of follow-up will grow. He encouraged the reporting of data into REDCAP. A number of countries in $\mathrm{A}-\mathrm{CaP}$ are now using Enzalutamide and pharmaceutical companies may be very interested in supporting the study. It would not be an unreasonable request to ask such companies for funding.

Dr Akaza noted that Dr Lim's presentation showed the tremendous potential for the A-CaP data. He encouraged all members to utilize the data.

Dr Carroll noted that the A-CaP study has developed tremendously over the last year and that it would be extremely interesting going forward.

Dr Carlile reported on incidence of Hawaiian prostate cancer, noting that the majority of cases are accounted for by Caucasian and Japanese Americans. The most used treatment was surgery and watchful waiting is also often used. Incidence has declined slightly in the past 3 years and the majority of prostate cancer is localized.

\section{Funding}

The Malaysian delegation expressed their sincere thanks to Malaysian NIH (National Institute of Health), Malaysian Ministry of Health for assisting this A-CaP study by the Grant number of NMRR-16-1340-31177.

\section{Conflict of interest statement}

This study was partly supported by research funds from Takeda Pharmaceutical Company Limited and Astellas Pharma Inc. These sponsors had no control over the interpretation, writing, or publication of this work.

\section{Appendix}

The authors' names are continued below following from the first page.
Jason L. Letran ${ }^{16}$, Edmund Chiong ${ }^{17}$, Tong-lin Wu ${ }^{18}$, Bannakij Lojanapiwat $^{19}$, Seung Hwan Lee ${ }^{20}$, Levent Türkeri ${ }^{21}$, Ghazi M. Al Edwan $^{22}$, Vu Le Chuyen ${ }^{23}$, Matthew Cooperberg ${ }^{24,25}$, Peter Carroll ${ }^{26}$, Robert Carlile ${ }^{27}$, Yoshihiko $\mathrm{Hirao}^{28}$, Shigeo $\mathrm{Horie}^{29}$, Mizuki Onozawa $^{30}$, Yasuhide Kitagawa ${ }^{31}$, Mikio Namiki ${ }^{32}$, Kazuhiro Suzuki $^{33}$, Takashi Fukagai ${ }^{34}$, Jun Miyazaki ${ }^{35}$ and Atsushi Mizokami ${ }^{36}$

${ }^{16}$ Department of Urology, Cardinal Santos Medical Center, Manila, Philippines

${ }^{17}$ Department of Urology, University Surgical Cluster, National University Health System (NUHS), Singapore

${ }^{18}$ Department of Surgery, Kaohsiung Veterans General Hospital, Taiwan, China

${ }^{19}$ Department of Surgery, Faculty of Medicine, Chiang Mai University, Thailand

${ }^{20}$ Department of Urology, Severance Hospital, Yonsei University School of Medicine, Seoul, South Korea

${ }^{21}$ Department of Urology, Marmara University School of Medicine, Istanbul, Turkey

${ }^{22}$ Chairman of Special Surgery Department, Consultant Urologist JHU, KHCC Urology and Laparoscopic Urology Fellowship-Australia, Jordan

${ }^{23}$ Chief of Department of Urology, Binh Dan Hospital, Hochiminh City, Vietnam

${ }^{24}$ Department of Urology, School of Medicine, University of California San Francisco, San Francisco, CA, USA

${ }^{25}$ Department of Epidemiology \& Biostatistics, School of Medicine, University of California San Francisco, San Francisco, CA, USA

${ }^{26}$ Department of Urology and Epidemiology \& Biostatistics, School of Medicine, University of California San Francisco, CA, USA

${ }^{27}$ Department of Surgery, University of Hawaii, HI, USA

${ }^{28}$ Osaka Gyoumeikan Hospital, Osaka, Japan

${ }^{29}$ Department of Urology, Juntendo University Graduate School of Medicine, Tokyo, Japan

${ }^{30}$ School of Medicine, International University of Health and Welfare, Japan

${ }^{31}$ Komatsu Municipal Hospital, Japan

${ }^{32}$ Department of Urology, Hasegawa Hospital, Toyama, Japan

${ }^{33}$ Department of Urology, Gunma University Graduate School of Medicine, Japan

${ }^{34}$ Department of Urology, Showa University Koto Toyosu Hospital, Tokyo, Japan

${ }^{35}$ School of Medicine, International University of Health and Welfare, Japan

${ }^{36}$ Department of Integrative Center Therapy and Urology, Graduate School of Medical Science, Kanazawa University, Japan

\section{References}

1. Akaza H, Hirao Y, Kim CS, Oya M, Ozono S, Ye D, et al. Asia Prostate Cancer Study (A-CaP) launch symposium. Prostate Int 2016;4:88-96.

2. Kim XS, Lee JY, Chung BH, Kim WJ, Ng CF, et al. Report of the second Asian Prostate Cancer (A-CaP) study meeting. Prostate Int 2017;5: 95-103.

3. Lojanapiwat B, Youl Lee J, Gang Z, Kim C-S, Chi Fai N, Hakim L, et al. Report of the third Asian Prostate Cancer study meeting. Prostate Int 2018;1-8. https://doi.org/10.1016/j.prnil.2018.06.001. 